\title{
Early superior mesenteric artery revascularization for acute type A aortic dissection with cardiac tamponade and mesenteric malperfusion
}

\author{
Sho Kusadokoro ${ }^{1}$, Naoyuki Kimura ${ }^{1}$, Kosuke Miyoshi ${ }^{1}$, Daijiro Hori ${ }^{1}$, Manabu Shiraishi ${ }^{1}$, \\ and Atsushi Yamaguchi ${ }^{1}$ \\ ${ }^{1}$ Saitama Medical Center, Jichi Medical University
}

April 28, 2020

\begin{abstract}
We report herein successful treatment of a case of acute type A aortic dissection complicated by cardiac tamponade and mesenteric malperfusion. The patient was a 60-year-old man, with back and abdominal pain and in shock, who was transported to our hospital 2 hours after symptom onset. Computed tomography revealed DeBakey type I dissection with massive hemopericardium and obstruction of both the celiac artery and superior mesenteric artery. After emergency pericardiotomy and removal of the hematoma, superior mesenteric artery-external iliac artery bypass was constructed with a vein graft, and this restored mesenteric perfusion. Open distal hemiarch replacement was then performed. The postoperative course was uneventful. Superior mesenteric artery revascularization achieved immediately after release of the cardiac tamponade prevented further mesenteric ischemia and paved the way for the aortic repair.
\end{abstract}

\section{Keywords}

acute type A aortic dissection, cardiac tamponade, mesenteric malperfusion

\section{INTRODUCTION}

Acute type A aortic dissection (ATAAD) remains a life-threatening emergency. Cardiac tamponade ${ }^{1}$ and mesenteric malperfusion $^{2}$ are known to increase the operative risk in patients with ATAAD. Central aortic repair involving resection of the primary entry tear can improve the perfusion status by increasing blood flow in the true lumen. However, timely reperfusion is mandatory for organ protection, and the beneficial effects of central aortic repair are limited in patients with dissection extending to the peripheral aortic branch. Although the utility of endovascular stenting to clear arterial blockage has been reported, ${ }^{3}$ concomitant cardiac tamponade may further complicate the therapeutic process. We report herein successful treatment of a patient with ATAAD presenting with shock caused by cardiac tamponade and mesenteric malperfusion.

\subsection{Patient profile}

The patient was a 60-year-old man with hypertension and diabetes who was transported by ambulance to our hospital's emergency 2 hours after onset of sudden back and abdominal pain. On admission, the patient's blood pressure was $90 / 66 \mathrm{mmHg}$, and palpation revealed abdominal tenderness. Cardiac ultrasonography revealed preserved left ventricular wall motion, mild aortic insufficiency, and massive pericardial effusion. Contrast-enhanced computed tomography $(\mathrm{CT})$ revealed a primary entry tear in the ascending aorta, pericardial effusion, a patent false lumen and narrowed true lumen in the descending aorta, obstruction of the proximal celiac artery and superior mesenteric artery (SMA), and dissection extending into the right external iliac artery (Figure 1). Arterial blood gas analysis revealed metabolic acidosis ( $\mathrm{pH} 7.22)$, and the 
blood lactate level was increased to $7.46 \mathrm{mmol} / \mathrm{L}$. ATAAD (DeBakey type I) with cardiac tamponade and mesenteric malperfusion was diagnosed

\subsection{Surgical procedure}

The patient was transferred immediately to the operating room. During induction of anesthesia, the patient's blood pressure dropped to $55 / 35 \mathrm{mmHg}$. Median sternotomy and pericardiostomy were performed immediately. A massive hematoma was removed, improving the patient's hemodynamic status. Careful inspection revealed no active bleeding from the aorta. Exploratory laparotomy showed extensive bowel ischemia, recognized by intestinal pallor, absence of peristaltic movement, and loss of pulsation of the main trunk and branches of the SMA. To prevent progression to irreversible bowel damage, SMA-left external iliac artery bypass was performed by application of a saphenous vein graft. After revascularization of the SMA, mesenteric perfusion quickly improved, as evidenced by pulsation of the intestinal arteries and peristalsis. Cardiopulmonary bypass (CPB) was then established by cannulation of the right femoral artery and the superior and inferior vena cava and placement of a left ventricular venting tube via the right upper pulmonary vein. The patient was cooled to $21.9^{\circ} \mathrm{C}$, and the dissected ascending aorta was excised. The primary entry tear was resected, and open distal hemiarch replacement was performed. There were no further signs of mesenteric malperfusion during the surgery. The abdomen was closed on postoperative day (POD) 1 , and the patient was extubated on POD 2. The postoperative course was uneventful, and CT performed on POD 7 showed an expanded true lumen, opened side branches of the abdominal aorta, and patency of the venous graft. The patient was discharged complication free on POD 20. CT performed 8 months after the surgery revealed a patent venous graft (Figure 2). Twelve months have passed since the surgery, and the patient remains healthy.

\section{DISCUSSION}

Outcomes of aortic repair for ATAAD have improved. According to a Japanese Association for Thoracic Surgery annual report, overall in-hospital mortality of patients who underwent aortic repair for ATAAD in 2015 was $9.6 \%(468 / 4875) .{ }^{4}$ Organ malperfusion associated with ATAAD remains a surgical challenge; patient-specific approaches are required. Shiiya et al. reported that sole central aortic repair failed to revascularize the abdominal organ(s) in 5 of 6 patients with aortic branch dissection. ${ }^{5}$ Uchida et al. reported favorable outcomes of a surgical strategy that prioritizes peripheral revascularization in patients with organ malperfusion. ${ }^{6}$ If a patient shows stable hemodynamics and branch-type malperfusion, early revascularization seems to be a feasible approach. Our patient's ATAAD was complicated by severe mesenteric malperfusion resulting from occlusion of both the celiac artery and SMA. We think that sole central aortic repair or delayed SMA revascularization could have resulted in irreversible mesenteric ischemia.

Previously, we reported ATAAD complicated by malperfusion of at least one organ in 30.9\% (308/1029) of patients and that we found obesity (body mass index $>30 \mathrm{~kg} / \mathrm{m}^{2}$ ), preoperative shock (systolic blood pressure $<80 \mathrm{mmHg}$ ), and mesenteric malperfusion to be independent predictors of-in-hospital death for those with such malperfusion. ${ }^{7}$ Treatment of the ATAAD-induced cardiac tamponade is of paramount importance in patients undergoing immediate aortic repair; however, the optimal management technique remains controversial. Lin et al. reported similar outcomes between emergency subxiphoid pericardiotomy and emergency establishment of CPB. ${ }^{8}$ Pericardiotomy performed initially for hematoma removal poses a risk of continuous bleeding in cases of aortic rupture. Our case was complicated by cardiac tamponade leading to hemodynamic instability, but aortic rupture had not occurred. In cases of aortic rupture, CPB must be established promptly, before the aortic repair. Revascularization of abdominal organs would be the second priority in such cases.

In conclusion, in our case of ATAAD complicated by cardiac tamponade and severe mesenteric malperfusion, initial release of pericardial hematoma and revascularization of the SMA prevented further mesenteric ischemia and paved the way for aortic repair.

\section{Author contributions}


SK wrote the original draft of this report, and NK revised it. KM, DH, MS and AY were involved in the patient's care. All authors have reviewed and approved the submission of the manuscript.

\section{REFERENCES}

1. Gilon D, Mehta RH, Oh JK, et al. Characteristics and in-hospital outcomes of patients with cardiac tamponade complicating type A acute aortic dissection. Am J Cardiol. 2009;103:1029-1031.

2. Di Eusanio M, Trimarchi S, Patel HJ, et al. Clinical presentation, management, and short-term outcome of patients with type A acute dissection complicated by mesenteric malperfusion: observations from the International Registry of Acute Aortic Dissection. J Thorac Cardiovasc Surg. 2013;145:385-390 e1.

3. Obied HY, Ghoneim A, Ibrahim MF. Hybrid approach to repair type A aortic dissection: combined endovascular superior mesenteric artery stenting and Bentall procedure. Aorta. 2014;2:156-160.

4. Association for Thoracic Surgery. Committee for Scientific Affairs, The Japanese Association for Thoracic Surgery; Masuda M, Endo S, Natsugoe S, et al. Thoracic and cardiovascular surgery in Japan during 2015: annual report by The Japanese Association for Thoracic Surgery. Gen Thorac Cardiovasc Surg. 2018:66, 581-615.

5. Shiiya N, Matsuzaki K, Kunihara T, Murashita T, Matsui Y. Management of vital organ malperfusion in acute aortic dissection: proposal of a mechanism-specific approach. Gen Thorac Cardiovasc Surg. 2007;55:8590.

6. Uchida K, Karube N, Kasama K, et al. Early reperfusion strategy improves the outcomes of surgery for type A acute aortic dissection with malperfusion. J Thorac Cardiovasc Surg. 2018;156:483-489.

7. Kawahito K, Kimura N, Yamaguchi A, Aizawa K. Malperfusion in type A aortic dissection: results of emergency central aortic repair. Gen Thorac Cardiovasc Surg. 2019;67:594-601.

8. Lin CY, Wu MY, Tseng CN, et al. Surgical rescues for critical hemopericardium complicated by acute type A aortic dissection: emergent subxiphoid pericardiotomy or cardiopulmonary bypass first? PloS One. 2020;15:e229648.

Figure Legends

\section{FIGURE 1}

CT images obtained preoperatively depict (A) an entry tear in the ascending aorta, a patent false lumen with a narrowed rue lumen in the descending aorta, and massive pericardial effusion; (B,C) obstruction of the proximal abdominal aortic branches, i.e., celiac artery and SMA; (D) dissection extending into the right external iliac artery but an intact left external iliac artery; and (E) obstruction of the proximal celiac artery and SMA (3D CT image). CT, computed tomography; SMA, superior mesenteric artery.

\section{FIGURE 2}

3D CT image obtained 8 months after the surgery showing the vein graft from the left external iliac artery to the SMA to be patent, with blood flowing freely through the celiac artery and SMA. CT, computed tomography; SMA, superior mesenteric artery. 


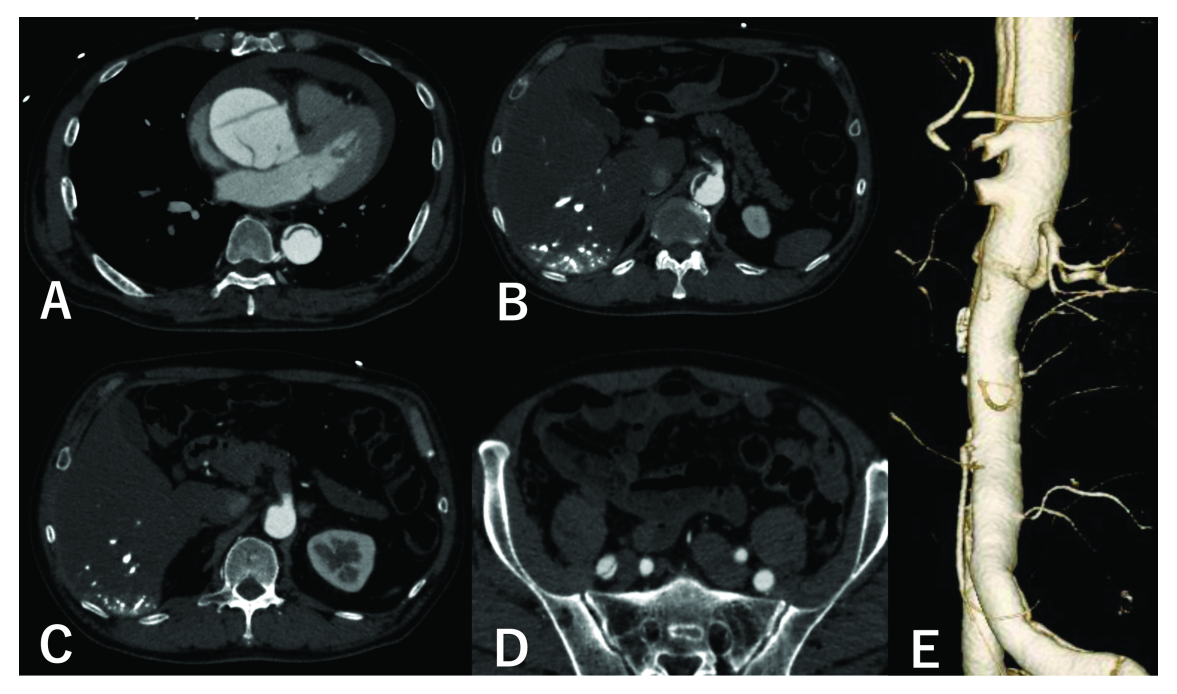




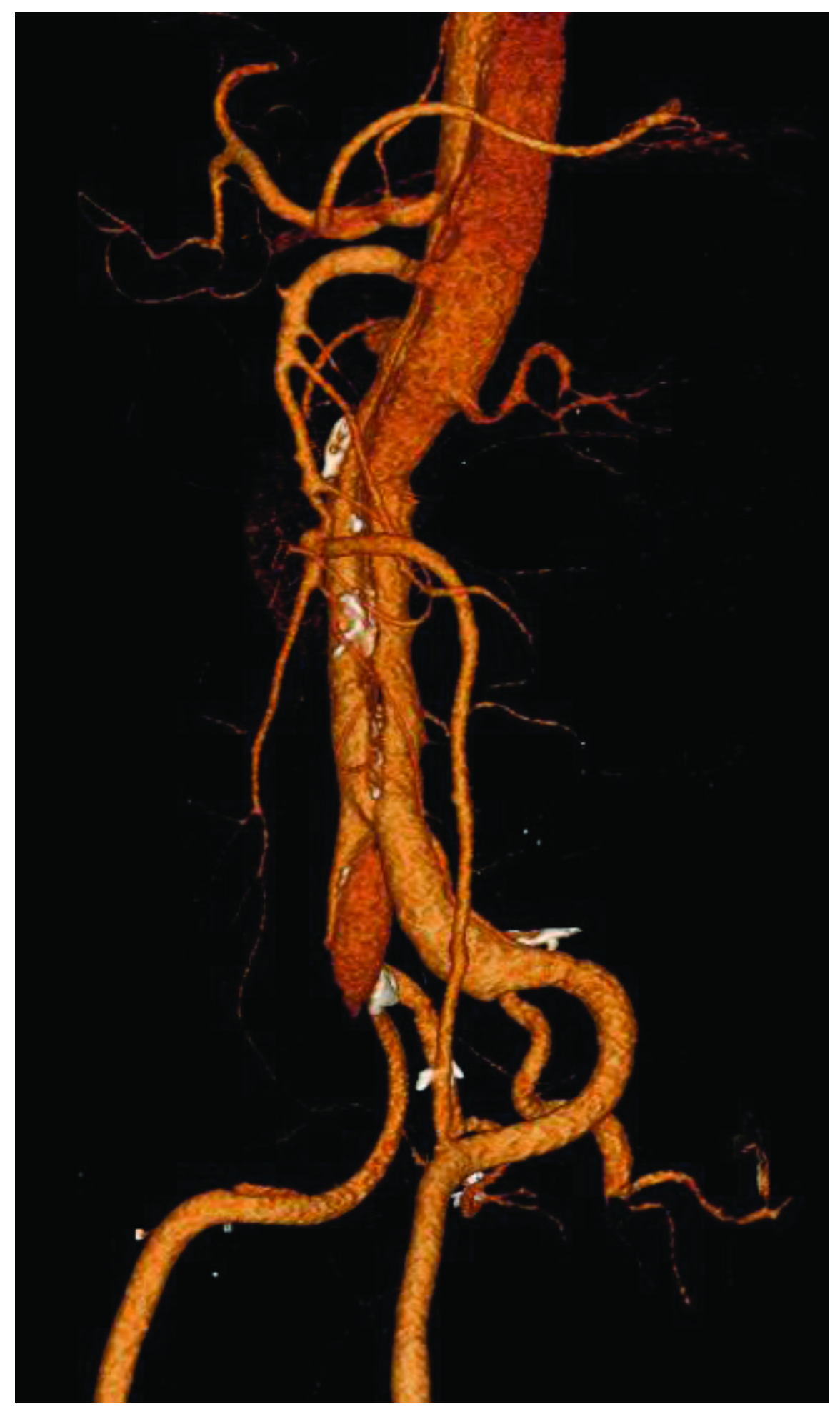

